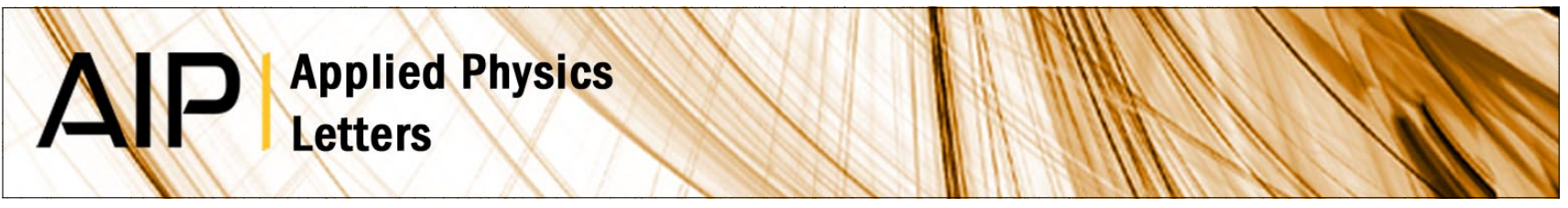

\title{
Formation of an extended CoSi2 thin nanohexagons array coherently buried in silicon single crystal
}

Guinther Kellermann, Luciano A. Montoro, Lisandro J. Giovanetti, Paula C. dos Santos Claro, Liang Zhang et al.

Citation: Appl. Phys. Lett. 100, 063116 (2012); doi: 10.1063/1.3683493

View online: http://dx.doi.org/10.1063/1.3683493

View Table of Contents: http://apl.aip.org/resource/1/APPLAB/v100/i6

Published by the American Institute of Physics.

Additional information on Appl. Phys. Lett.

Journal Homepage: http://apl.aip.org/

Journal Information: http://apl.aip.org/about/about_the_journal

Top downloads: http://apl.aip.org/features/most_downloaded

Information for Authors: http://apl.aip.org/authors

\section{ADVERTISEMENT}
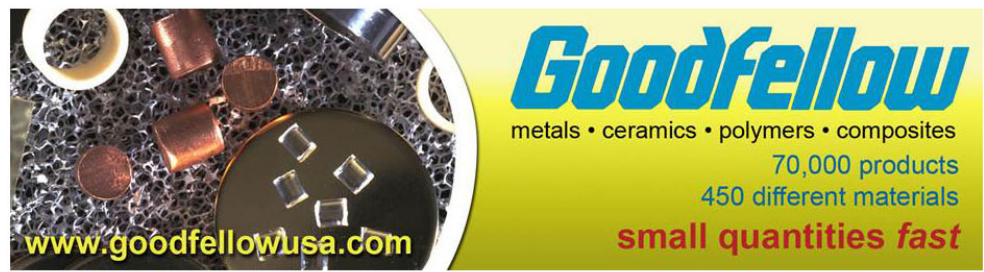


\title{
Formation of an extended $\mathrm{CoSi}_{2}$ thin nanohexagons array coherently buried in silicon single crystal
}

\author{
Guinther Kellermann, ${ }^{1}$ Luciano A. Montoro, ${ }^{2}$ Lisandro J. Giovanetti, ${ }^{3, a)}$ \\ Paula C. dos Santos Claro, ${ }^{3}$ Liang Zhang, ${ }^{4, b)}$ Antonio J. Ramirez, ${ }^{2}$ Félix G. Requejo, ${ }^{3}$ \\ and Aldo F. Craievich ${ }^{5}$ \\ ${ }_{1}^{1}$ Departamento de Física, Universidade Federal do Paraná, Caixa Postal 19091, Curitiba, Paraná 81531-990, \\ Brazil \\ ${ }^{2}$ Brazilian Nanotechnology National Laboratory (LNNano), Caixa Postal 6192 - CEP 13083-970, Campinas, \\ São Paulo 13083-100, Brazil \\ ${ }^{3}$ Instituto de Investigaciones Fisicoquímicas Teóricas y Aplicadas (INIFTA, CONICET, \\ Departamento de Química, Facultad de Ciencias Exactas, Universidad Nacional de La Plata), CC/16 suc. 4, \\ 1900 La Plata, Argentina \\ ${ }^{4}$ School of Chemical, Biological and Materials Engineering, University of Oklahoma, \\ Norman Oklahoma 73019, USA \\ ${ }^{5}$ Institute of Physics, University of Sao Paulo, CP 66318, CEP 05315-970, São Paulo, Brazil
}

(Received 21 December 2011; accepted 12 January 2012; published online 10 February 2012)

\begin{abstract}
A Co-doped silica film was deposited on the surface of a $\mathrm{Si}(100)$ wafer and isothermally annealed at $750^{\circ} \mathrm{C}$ to form spherical Co nanoparticles embedded in the silica film and a few atomic layer thick $\mathrm{CoSi}_{2}$ nanoplatelets within the wafer. The structure, morphology, and spatial orientation of the nanoplatelets were characterized. The experimental results indicate that the nanoplatelets exhibit hexagonal shape and a uniform thickness. The $\mathrm{CoSi}_{2}$ nanostructures lattice is coherent with the Si lattice, and each of them is parallel to one of the four planes belonging to the $\{111\}$ crystallographic form of the host lattice. () 2012 American Institute of Physics. [doi:10.1063/1.3683493]
\end{abstract}

The ever-growing demand of methods for the fabrication of nanostructured materials is one of the most exciting challenges in modern materials science and nanotechnology. Self-assembly and lithography techniques-including those using synthetic or biological templates-are paradigms for these $\operatorname{architectures}^{1,2}$ that can lead to commercial applications of nanoelectronics ${ }^{3}$ for the development, for example, of sensors ${ }^{4}$ and magneto-optical devices. ${ }^{5}$ On the other hand, effective large-scale integration of such nano-objects needs high-throughput strategies. In this context, reproducible, controlled, and clean alternatives for bottom-up growth of nanostructured materials over large areas are nowadays highly relevant issues.

Transition metal silicides such as $\mathrm{TiSi}_{2}, \mathrm{CoSi}_{2}$, and NiSi have been extensively studied during the past decade due to their importance as contact materials for microelectronic devices. Since $\mathrm{CoSi}_{2}$ exhibits high electrical conductivity and good thermal stability, this material has attracted the attention of many researchers because it is a good candidate for nanocomponents requiring high electrical conductivity. ${ }^{6}$ Furthermore and due to the low mismatch $(-1.2 \%)$ with respect to the $\mathrm{Si}$ lattice, $\mathrm{CoSi}_{2}$ films can be epitaxially grown on the surface of $\mathrm{Si}(111)$ wafers, thus displaying attractive properties for potential applications in high-speed devices.

Unfortunately, the classical processes for polycrystalline $\mathrm{CoSi}_{2}$ growth have serious drawbacks such as agglomeration, silicon consumption, and leakage current. Clearly,

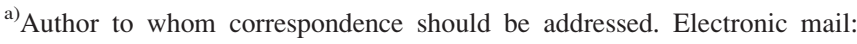
lisandro@fisica.unlp.edu.ar.

${ }^{b}$ Present address: Pacific Northwest National Laboratory, Richland, Washington 99352, USA
}

agglomeration becomes critical for ultra-large scale integration in nanotechnology because of the limitation in line width and shallowness source/drain junction. Moreover, agglomeration induces the formation of large $\mathrm{CoSi}_{2}$ grains and consequently to resistance degradation. ${ }^{7,8}$

The specific type of Co-Si compounds that develops during the growth processes is also a relevant issue because the different phases of the equilibrium phase diagram of this binary system exhibit quite different resistivities, namely $10-20,110,140$, and $20 \mu \Omega / \mathrm{cm}$ for $\mathrm{Co}, \mathrm{Co}_{2} \mathrm{Si}, \mathrm{CoSi}$, and $\mathrm{CoSi}_{2}$, respectively. ${ }^{9}$ Previous investigations established that the silicide phase nucleates locally on structural defects of the Si surface during an exothermic reaction, this first step being followed by a self-ordered lateral growth. ${ }^{10-19}$ Wang et $a l^{20}{ }^{20}$ reported the fabrication of micro/nanoscale pits with facile shape, orientation, and size controls on a Si surface via an Au-nanoparticles-assisted vapor transport method. The pit dimensions can be continuously tuned from $70 \mathrm{~nm}$ up to several $\mu \mathrm{m}$. Triangles, squares, and wire/hexagons morphologies were detected in $\mathrm{Si}(111), \mathrm{Si}(001)$, and $\mathrm{Si}(110)$ substrates, respectively.

Here, we describe a simple, controlled, and reproducible procedure for obtaining thin and non-agglomerated $\mathrm{CoSi}_{2}$ nanoplatelets coherently grown inside a silicon (001) single crystal. A precise structural characterization of the $\mathrm{CoSi}_{2}$ thin structures was performed by using transmission electron microscopy (TEM) and grazing incidence small angle $\mathrm{X}$-ray scattering (GISAXS) techniques.

Surface of a $\operatorname{Si}(001)$ substrate $\left(2 \times 1 \mathrm{~cm}^{2}\right)$ was cleaned with ethanol and dried by blowing $\mathrm{N}_{2}$ prior to depositing a $\mathrm{SiO}_{2}$-based thin film. To obtain the Co impregnated $\mathrm{SiO}_{2}$ thin film, a precursor solution was prepared by dissolving 
cobalt(II) nitrate $(11 \mu \mathrm{mol} / \mathrm{g})$ and tetraethyl orthosilicate (TEOS, $240 \mu \mathrm{mol} / \mathrm{g}$ ) in isopropanol. The $\mathrm{pH}$ of the aforementioned solution was then adjusted to 2-3 with $\mathrm{HCl}$. A volume of $8 \mu \mathrm{L}$ of such solution was dropped to the center of the cleaned silicon wafer sitting in a Petri dish. A lid was then put on the top of the dish to let the solvent to evaporate slowly and uniformly. After $1 \mathrm{~h}$, the resulting still wet deposited film was dried during $10 \mathrm{~min}$ at $100^{\circ} \mathrm{C}$ and calcined in air during $15 \mathrm{~min}$ at $500^{\circ} \mathrm{C}$.

Subsequently, the sample was reduced under hydrogen flow $(500 \mathrm{sccm})$ for $1 \mathrm{~h}$ at $500^{\circ} \mathrm{C}$. After this step, the oven was purged using a He flux (1000 sccm), while the temperature was increased up to $750^{\circ} \mathrm{C}$ at $10^{\circ} \mathrm{C} / \mathrm{min}$. The sample was finally kept $1 \mathrm{~h}$ under these conditions to promote the diffusion of Co atoms and to approach the thermodynamic equilibrium. The oven was then let to cool down to room temperature under He flow.

The thermal treatment for $1 \mathrm{~h}$ at $500{ }^{\circ} \mathrm{C}$ in $\mathrm{H}_{2}$ atmosphere was applied in order to promote the chemical reduction to metallic Co state of the as impregnated cobalt nitrate. A further heat treatment for $1 \mathrm{~h}$ at $750^{\circ} \mathrm{C}$ induced the up-hill diffusion of $\mathrm{Co}$ atoms and their precipitation as metallic Co nanoparticles inside the $\mathrm{SiO}_{2}$ film volume or deposited on the $\mathrm{Si}(001)$ surface. In addition to the expected clustering of Co atoms inside the $\mathrm{SiO}_{2}$ thin film, these atoms also penetrated into the $\mathrm{Si}(001)$ wafer to form buried thin $\mathrm{CoSi}_{2}$ nanoplatelets.

The initial step of our preparation procedure was to deposit a Co-doped $\mathrm{SiO}_{2}$ thin film onto a $\mathrm{Si}(001)$ flat substrate. The basic idea was to build up a Co-doped buffer layer and then to promote de diffusion of Co atoms into the Si wafer by a controlled thermal treatment.
Fig. 1(a) exhibits a TEM image obtained from a crosssectioned sample at [110] zone axis. TEM cross-section specimens with their faces normal to the $\mathrm{Si}[110]$ zone axis were prepared using manual and dimple polishing, followed by liquid nitrogen cooled $\mathrm{Ar}^{+}$ion-beam thinning with energies ranging between 3.5 and $2 \mathrm{keV}$, at incidence angles gradually decreasing from $7^{\circ}$ to $4^{\circ}$. The sample features result from a heat treatment of the $\mathrm{Si}(001)$ wafer covered with a homogeneously Co-doped silica film. This image shows the presence of small Co nanoparticles embedded in the silica thin film and also small nanoplatelets buried into the $\mathrm{Si}(001)$ single crystal. Fig. 1(b) displays a TEM image where two similar buried nanoplatelets can be observed. On the right a low-contrasted nanoplatelet exhibiting a hexagonal shape and on the left side another one in a lateral view with a rod-like form (both oriented at [110] zone axis). By using a sample tilting procedure, it is possible to demonstrate that both structures present the same form but with different growth planes and orientation (see supplementary Fig. S1 for images of tilted sample). ${ }^{25}$ Fig. 1(c) shows a front view image of a nanoplatelet, which clearly evidences the hexagonal shape of the buried nanostructures.

The analysis of a number of TEM images taken at different sample orientations indicated that the buried nanoplatelets exhibit four different orientations, with their large flat surfaces parallel to one of the four planes of the $\mathrm{Si}\{111\}$ crystallographic form. The nanoplatelets exhibit an overall 4-fold rotational symmetry around the $\mathrm{Si}[001]$ axis, normal to the silicon surface, as schematically shown in Fig. 1(d). Note that the hexagons are not rigorously regular. As depicted in Fig. 1(c), the nanoplatelets present a truncated shape at the side in contact with the silica/silicon interface. (a)

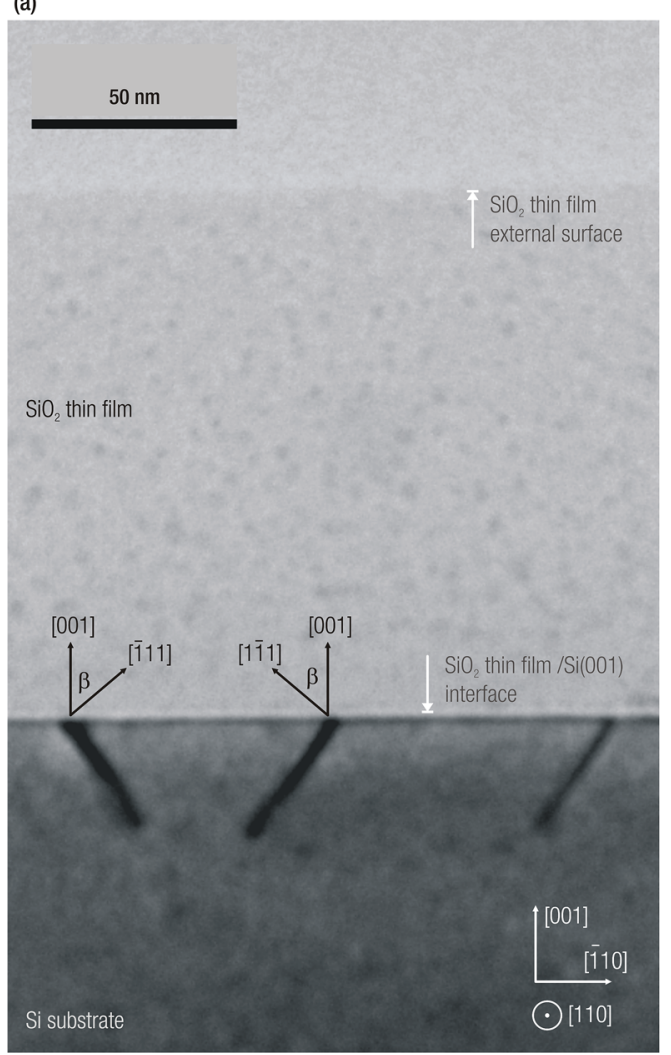

(b)

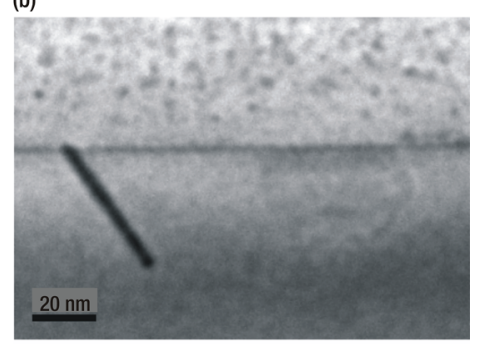

(c)

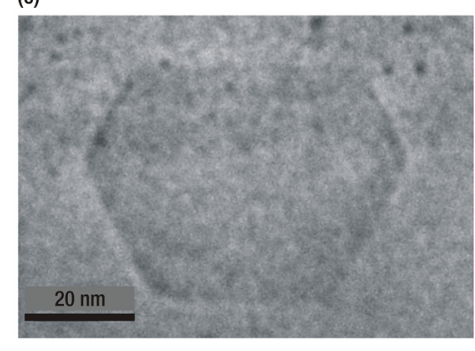

(d)

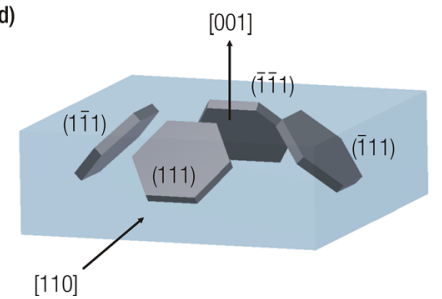

FIG. 1. (Color online) TEM images. (a) TEM image corresponding to a cross-sectioned (001) silicon wafer in which a Co-doped silica $\left(\mathrm{SiO}_{2}\right)$ was deposited. We can notice the presence of small Co nanoparticles embedded in the silica film (top) and three nanoplatelets buried in the $\mathrm{Si}(001)$ wafer with their large faces normal to planes corresponding to the $\mathrm{Si}\{111\}$ crystallographic form (bottom). (b) Image with two different nanoplatelets oriented at $\mathrm{Si}[110]$ zone axis. (c) Same specimen shown in (b) oriented along the large faces normal to the electron beam showing a projection of the hexagonal nanoplatelet onto the Si(110) plane. (d) Schematic display of the hexagonal $\mathrm{CoSi}_{2}$ nanoplatelets with four different orientations, each of them parallel to one plane belonging to the $\mathrm{Si}\{111\}$ crystallographic form. 
In order to establish the chemical composition of the nanoplatelets, TEM with x-ray energy dispersive spectroscopy (XEDS) analyses was performed by using a $3 \mathrm{~nm}$ electron probe. A JEM-2100 HTP TEM with a $\mathrm{LaB}_{6}$ electron gun was used at an accelerating voltage of $200 \mathrm{kV}$. The images were recorded by using CCD cameras. XEDS measurements were performed by using a $\mathrm{Si}-\mathrm{Li}$ detector coupled to the TEM. Complementary results of XEDS and high-resolution (HR) TEM analyses indicated that the composition and the structure of the buried nanoplatelets are those of the stoichiometric $\mathrm{CoSi}_{2}$ phase with a cubic $\mathrm{CaF}_{2}-$ like structure.

Fig. 2(a) shows a HRTEM image of a nanoplatelet in a specific defocus value. At this condition, the high-intensity peaks can be directly related to the atomic column positions, as supported by HRTEM image simulation. Fig. 2(b) indicates the atomic position at the interfaces $\mathrm{Si}_{\mathrm{CoSi}}: \mathrm{Si}$ in a magnified region from Fig. 2(a). This atomic model was verified by HRTEM image simulation and indicates the occurrence of a twined interface between the $\mathrm{Si}: \mathrm{CoSi}_{2}$ phases. These $\mathrm{Si}^{-\mathrm{TMSi}_{2}}(\mathrm{TM}=$ transition metal $)$ interface

(a)

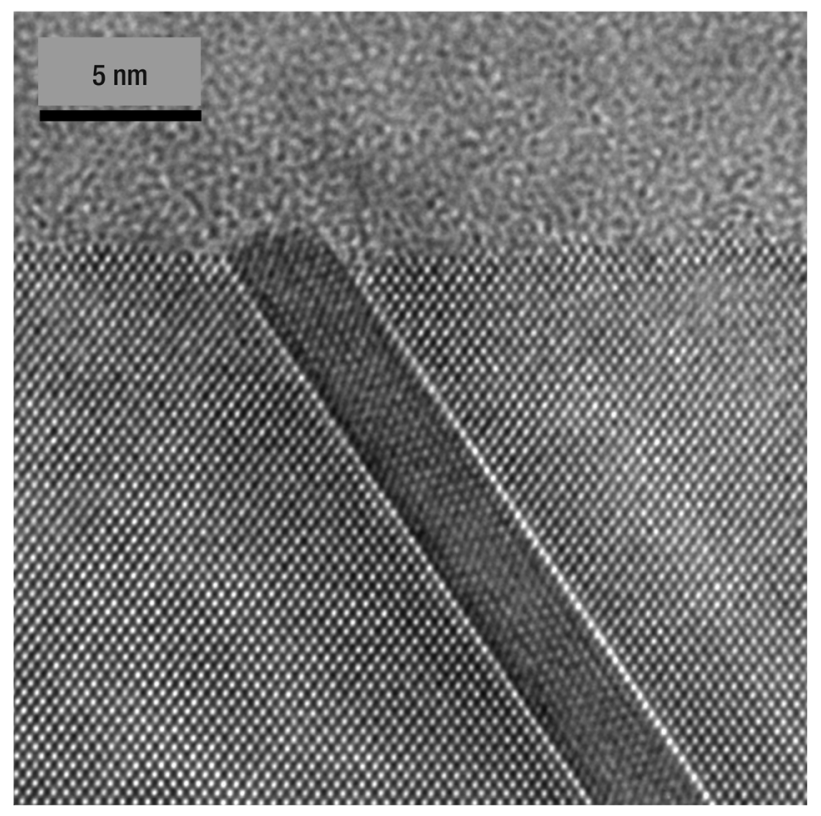

(b)

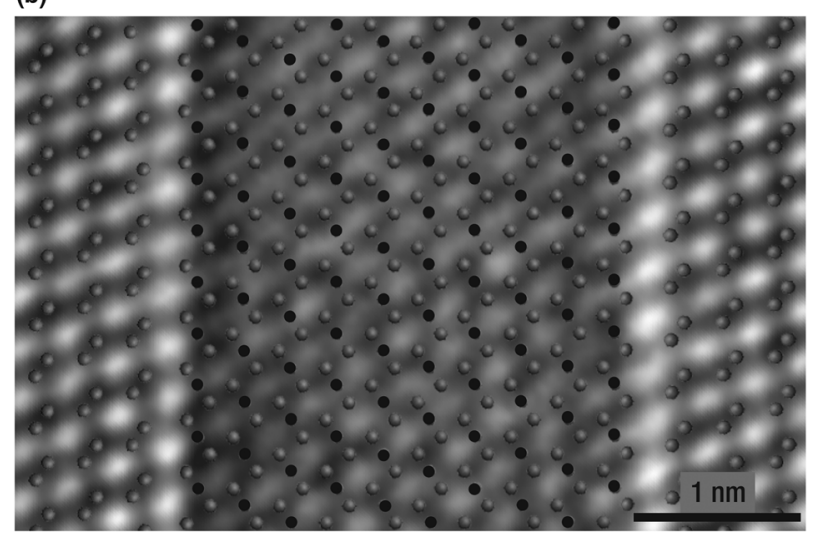

FIG. 2. HRTEM images. (a) HRTEM image of $\mathrm{CoSi}_{2}$ nanoplatelets buried in a $\mathrm{Si}(001)$ single crystal and parallel to one plane of the $\mathrm{Si}(111)$ crystallographic form. (b) Details of the atomic arrangement, in which grey and black circles correspond to a $\mathrm{Si}$ and $\mathrm{Co}$ atoms position, respectively. structures have been extensively studied by different authors. ${ }^{21,22}$ From these studies, it is possible to attribute an 8B-type interface structure. This means that the transition metal at the interface is in an 8-fold coordination with the $\mathrm{Si}$ atoms, and the silicide is rotated by $180^{\circ}$ about the [111] Si axis (a twined structure).

A quantitative analysis of our TEM images indicated that the $\mathrm{CoSi}_{2}$ nanoplatelets have a homogeneous thickness $\mathrm{t}=(3.17 \pm 0.23) \mathrm{nm}$ and an average length of the hexagon sides $d=(24 \pm 3) \mathrm{nm}$. The crystal lattice of the cubic $\mathrm{CaF}_{2^{-}}$ like structure of the $\mathrm{CoSi}_{2}$ nanoplatelets exhibits a low lattice mismatch with respect to the cubic lattice of the $\mathrm{Si}$ diamond-like structure $(\Delta \mathrm{a} / \mathrm{a}=1.2 \%$, a being the lattice parameter). This feature favors a particular process of growth during which the crystal lattice of the $\mathrm{CoSi}_{2}$ nanoplatelets establishes and maintains its coherence with respect to the host Si lattice, as can be clearly seen by HRTEM in Figs. 2(a) and 2(b).

In order to achieve a global characterization of the nano-objects individually observed in TEM images, we have additionally GISAXS experiments performed at the XRD2 $\mathrm{x}$-ray diffraction beam line of the Brazilian Synchrotron Light Laboratory (LNLS), Campinas, Brazil, using a 0.1612 $\mathrm{nm}$ wavelength beam. The two-dimensional patterns of the GISAXS intensity were recorded by using a $10 \times 10 \mathrm{~cm}^{2}$ imaging plate $2 \mathrm{D}$ detector with a $10^{6}$ dynamical range and a $0.2 \times 0.2 \mathrm{~mm}^{2}$ pixel size, following the geometry proposed by Salditt et al. ${ }^{23}$ In this geometry, the incidence angle of the $\mathrm{x}$-ray beam on the sample external surface is kept constant, and the $2 \mathrm{D}$ scattering intensity is measured using an imaging-plate or 2D photon detector. For additional description of the GISAXS procedures, analysis, and modeling, see supplementary information. ${ }^{25}$ The shape and size distribution of the $\mathrm{CoSi}_{2}$ nano-hexagons buried in the $\mathrm{Si}$ wafer-together with their orientations averaged over a large sample volume-were analyzed over an irradiated area of about $3 \times 20$ $\mathrm{mm}^{2}$ (footprint of the $\mathrm{x}$-ray beam on the surface of the flat samples).

GISAXS intensity patterns were determined at different azimuthal rotation angles $\phi$ around the $\mathrm{Si}(001)$ axis-normal to the sample surface-allowing us to establish the orientation of the three-dimensional structure associated to the dispersers embedded in the Si substrate. The angle $\phi=0^{\circ}$ was chosen in such a way that the projection of the incoming $\mathrm{x}$ ray beam on $\mathrm{Si}(001)$ plane coincides with the $\mathrm{Si}[110]$ crystallographic axis (see Fig. 3(h) for angle reference). The incidence angle between the incoming beam and the surface of the sample $\alpha_{i}=0.3^{\circ}$ was selected in order to achieve an adequate value for the primary $\mathrm{x}$-ray beam penetration optimizing the scattering intensity.

Figs. 3(a) $-3(\mathrm{~g})$ display one among a series of GISAXS 2D images experimentally determined. These patterns correspond to the sample laterally oriented at different azimuthal angles. Most of those images exhibit two elongated scattering lobes, between -12 and $12^{\circ}$, with a maximum intensity at $0^{\circ}$, extending from the origin of the reciprocal space to rather large values of transferred momentum. The major axes of both elongated lobes make the same angle with the plane that contains the Si[110] crystallographic direction. Since this angle is approximately equal to the expected angle 


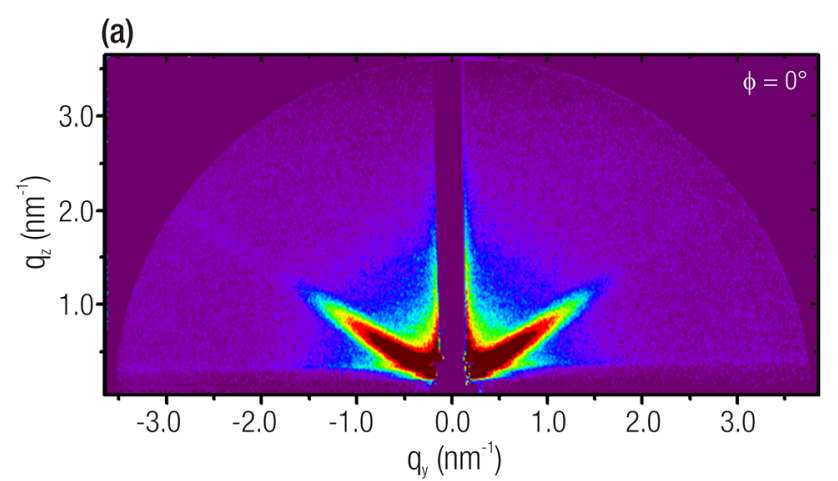

(b)

(c)

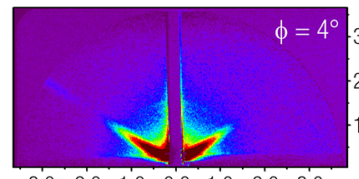

$\begin{array}{llllllll}3.0 & -2.0 & -1.0 & 0.0 & 1.0 & 2.0 & 3.0\end{array}$ $\mathrm{q}_{\mathrm{y}}\left(\mathrm{nm}^{-1}\right)$

(d)

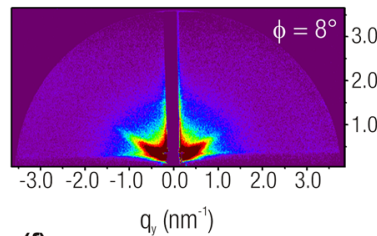

(f)

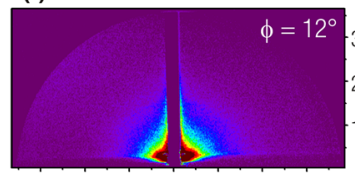

$\begin{array}{lllllll}-3.0 & -2.0 & -1.0 & 0.0 & 1.0 & 2.0 & 3.0\end{array}$ $\mathrm{q}_{\mathrm{y}}\left(\mathrm{nm}^{-1}\right)$

(h)

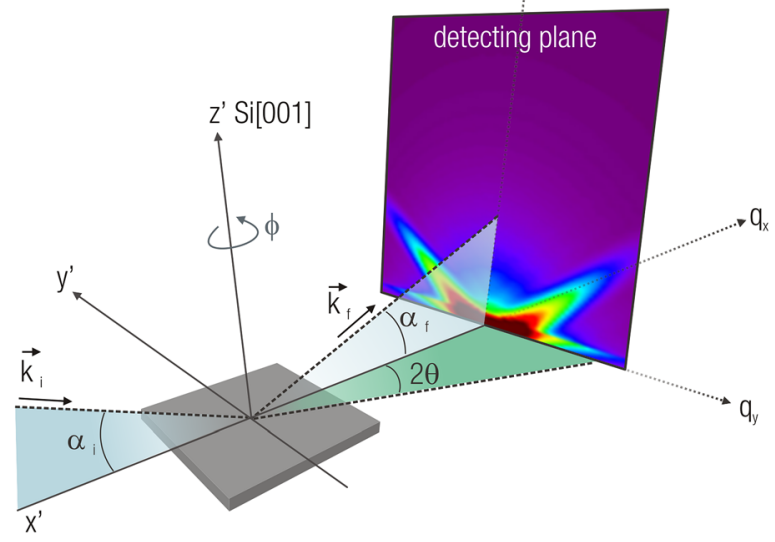

FIG. 3. (Color online) GISAXS results. Experimental 2D-GISAXS images taken for different azimuthal angles: (a) $\phi=0^{\circ}$, (b) $\phi=4^{\circ}$, (c) $\phi=-4^{\circ}$, (d) $\phi=8^{\circ}$, (e) $\phi=-8^{\circ}$, (f) $\phi=12^{\circ}$, and (g) $\phi=-12^{\circ}$, and (h) schematic picture showing the geometry used in the GISAXS experiments.

between the $\mathrm{Si}[110]$ and $\mathrm{Si}[111]$ crystallographic directions, i.e., equal to $35.3^{\circ}$, we could safely conclude that the main directions of the observed elongated scattering lobes shown in Fig. 3(a) are normal to two lattice planes belonging to the $\mathrm{Si}\{111\}$ crystallographic form. The lobe feature disappears when azimuthal angle increases up to $12^{\circ}$ (Fig. 3(d)). This same GISAXS pattern repeats every time the azimuthal angle is incremented by $90^{\circ}$, as is expected from the 4-fold rotational symmetry around $\mathrm{Si}[001]$ axis.
In addition to the GISAXS intensity produced by the hexagonal nanoplatelets, the isotropic scattering due to the uncorrelated set of spherical nanoparticles embedded in the $\mathrm{SiO}_{2}$ was also observed. Because of the quasi random spatial distribution of the spherical particles, their contribution to the total scattering intensity is expected to be isotropic.

Taking into account the preliminary conclusions from our GISAXS measurements mentioned above and the TEM analysis previously described, a modeled GISAXS function was calculated. This simulated function assumes that the scattering intensity is produced by (1) thin hexagonal $\mathrm{CoSi}_{2}$ nanoplatelets inserted in four different planes of the $\mathrm{Si}$ lattice corresponding to the $\{111\}$ crystallographic form and, additionally, (2) spherical Co nanoparticles without spatial correlation embedded in the silica thin film. A lognormal function was proposed to describe the radius distribution of the spherical Co nanoparticles, and same thickness ( $t$ ) and lateral sizes (d) were assumed for all $\mathrm{CoSi}_{2}$ nanohexagons.

Fig. 4(a) displays a 2D GISAXS pattern corresponding to the studied sample for an azimuthal angle $\phi=0$. The simulated 2D image displayed in Fig. 4(b) evidences a good qualitative agreement with the experimental pattern shown in Fig. 4(a). Experimental and modeled GISAXS profiles shown in Fig. 4(c)—corresponding to the different $\mathrm{q}_{\mathrm{z}}$ values (see Fig. 3(h)) —exhibit an excellent agreement. All relevant structural parameters of the studied material were determined as those of the simulated function that exhibited the best fit to the experimental results.

The best fit procedure yielded a thickness and a lateral side of the $\mathrm{CoSi}_{2}$ nanoplatelets buried into the $\mathrm{Si}$ wafer $\mathrm{t}=(2.5 \pm 0.3) \mathrm{nm}$ and $\mathrm{d}=(19.5 \pm 0.5) \mathrm{nm}$, respectively. The average radius of the spherical nanoparticles embedded in the silica thin film was established to be $<\mathrm{R}\rangle=(0.43 \pm 0.03) \mathrm{nm}$, and its relative standard deviation $\sigma_{\mathrm{R}} /<\mathrm{R}>$, being equal to $0.58 \pm 0.06$. The non-perfect symmetry at the curves in Fig. 4(c) is due to the non-centered position of the sample at the azimuthal angle, which was fitted in $1.5^{\circ}$.

In summary, the diffusion of Co atoms from silica thin films deposited on a $\mathrm{Si}(001)$ wafer induces the formation of thin hexagonal $\mathrm{CoSi}_{2}$ nanoplatelets embedded in the host $\mathrm{Si}$ single crystal. These buried structures exhibit four different orientations, each of them strictly parallel to one of the four planes of the $\mathrm{Si}\{111\}$ crystallographic form. The $\mathrm{CoSi}_{2}$ nanohexagons are parallel only to planes of the $\{111\}$ form, because the free energy of their (coherent) interface is in this case lower than in the case of nanoplatelets parallel to other dense planes of the Si lattice such as those of the $\{100\}$ or $\{110\}$ crystallographic forms. ${ }^{24}$

Relevant information of local features of the studied material was obtained by TEM and over a large sample volume by GISAXS. This joint use of TEM and GISAXS techniques provided complementary and essential information, both being indispensable for achieving a clear and detailed description of the studied nanostructured material. The orientations and the geometrical features of the nanoplatelets determined from both TEM and GISAXS analyses are equivalent. The slight differences in the thickness and lateral 
(a)
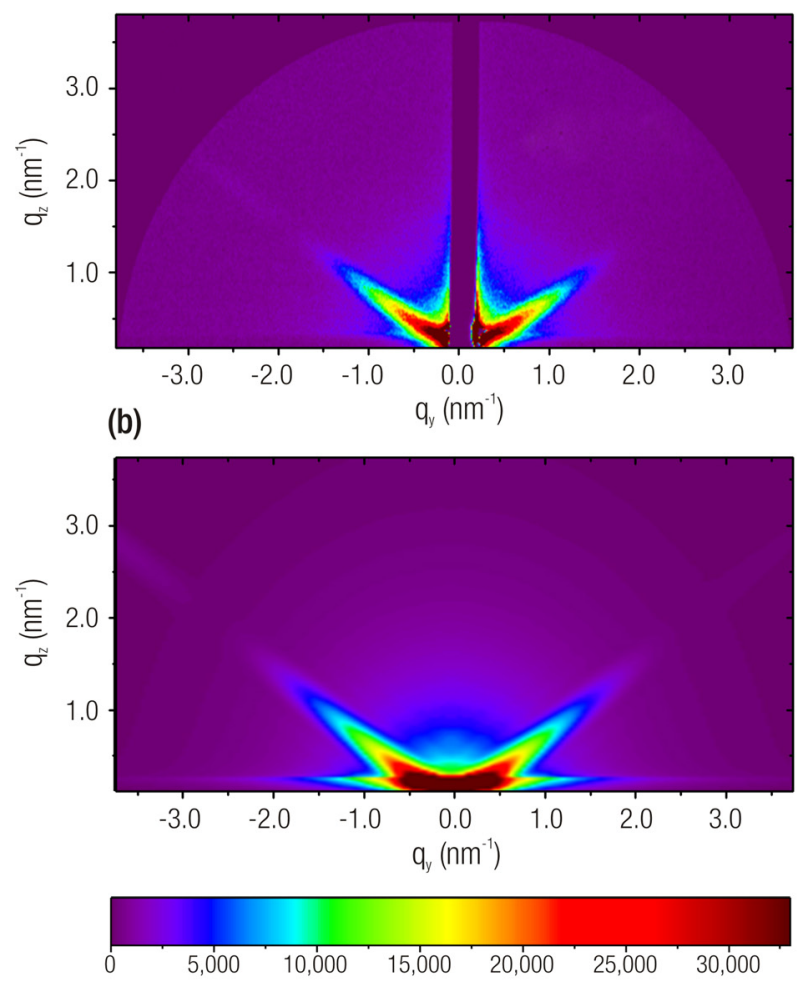

(c)

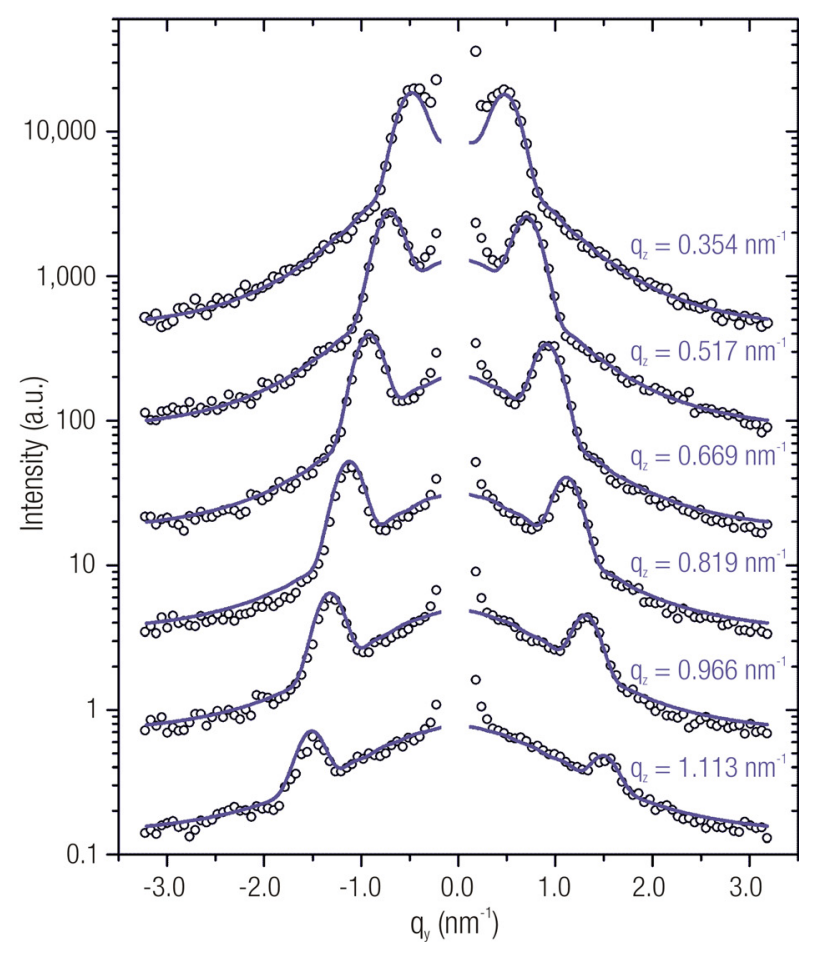

FIG. 4. (Color online) GISAXS modeling. (a) Experimental 2D GISAXS pattern corresponding angle $\phi=1.5$. The vertical dark ribbon is the shadow of a stopper that avoids to the azimuthal the strong totally reflected beam reaching the imaging plate. (b) Simulated 2D GISAXS pattern for which the best fit to the experimental pattern (a) was achieved. Below: GISAXS intensity color scale. (c) Experimental GISAXS 1D profiles (symbols) and fitted curves (continuous lines) corresponding to different constant $q_{z}$ values, i.e., different horizontal GISAXS linear profiles derived from the 2D image displayed in (a).

dimension of the nanohexagons determined by means of both techniques are a consequence of the low nanoplatelets sampling in TEM analysis.
Our results show that the process of diffusion of cobalt atoms from a cobalt-doped thin film into a $\mathrm{Si}(001)$ single crystalline wafer and the consequent formation of oriented $\mathrm{CoSi}_{2}$ nanoplatelets-coherently related to the crystallographic lattice of the host $\mathrm{Si}$ single crystal with well defined thickness and lateral sizes — can be achieved and well controlled.

Since the described process can be reproducibly achieved, it can also be considered as an original and simple alternative for the production of nanostructured materials with relevant physical properties, over a large area, which may find useful applications for developments of nano-integrated devices.

This work was partially supported by Brazilian Synchrotron Light Laboratory (LNLS), Brazilian Nanotechnology National Laboratory (LME-LNNano), CNPq and FAPESP, Brazil; ANPCYT (PICT 2008-00038) and CONICET (PIP 112-200801-03079), Argentina and CIAM collaborative project (CONICET/CNPq). The authors acknowledge Professor D. Resasco for fruitful discussions.

${ }^{1}$ Y. Huang, X. Duan, Q. Wei, and C. M. Lieber, Science 291, 630 (2001).

${ }^{2}$ C. L. Cheung, J. A. Camarero, B. W. Woods, T. Lin, J. E. Johnson, and J. J. De Yoreo, J. Am. Chem. Soc. 125, 6848 (2003).

${ }^{3}$ R. C. Chau, B. Doyle, S. Datta, K. Jack, and K. Zhang, Nature Mater. 6, 810 (2007).

${ }^{4}$ J. D. Caldwell, O. Glembocki, F. J. Bezares, N. D. Bassim, R. W. Rendell, M. Feygelson, M. Ukaegbu, R. Kasica, L. Shirey, and C. Hosten, ACS Nano 5, 4046 (2011).

${ }^{5}$ M. D. Kelzenberg, D. B. Turner-Evans, B. M. Kayes, M. A. Filler, M. C. Putnam, N. S. Lewis, and H. A. Atwater, Nano Lett. 8, 710 (2008).

${ }^{6}$ J. Derrien, M. De Crescenzi, E. Chainet, C. d' Anterroches, C. Pirri, G. Gewinner, and J. C. Peruchetti, Phys. Rev. B 36, 6681 (1987).

${ }^{7}$ S. Vaidya, S. P. Murarka, and T. T. Sheng, J. Appl. Phys. 58, 971 (1985).

${ }^{8}$ C. Y. Ting, F. M. d' Heurle, S. S. Iyer, and P. M. Fyrer, J. Electrochem. Soc. 133, 2621 (1986)

${ }^{9}$ F. Corni, R. Tonini, G. Ottaviani, S. Alberici, D. Erbetta, and T. Marangon, Microelectron. Eng. 76, 343 (2004).

${ }^{10}$ R. T. Tung, J. M. Gibson, and J. M. Poate, Appl. Phys. Lett. 42, 888 (1983).

${ }^{11}$ C. Pirri, J. C. Peruchetti, G. Gewinner, and J. Derrien, Phys. Rev. B 29, 3391 (1984).

${ }^{12}$ K. L. Wang, Solid State Technol. 28, 137 (1985).

${ }^{13}$ B. D. Hunt, N. Lewis, E. L. Hall, L. G. Turner, L. J. Schowalter, M. Okamoto, and S. Hashimoto, Mater. Res. Soc. Symp. Proc. 56, 151 (1985).

${ }^{14}$ F. A. D'Avitaya, S. Delage, E. Rosencher, and J. Derrien, J. Vac. Sci. Technol. B 3, 770 (1985).

${ }^{15}$ J. Henz, H. Von Känel, M. Ospelt, and P. Wachter, Surf. Sci. 189-190, 1055 (1987).

${ }^{16}$ N. Cherief, R. Cinti, M. De Crescenzi, J. Derrien, T. A. Nguyen Tan, and J. Y. Veuillen, Appl. Surf. Sci. 41-42, 241 (1989).

${ }^{17}$ M. Gomoyunova, I. Pronin, D. Valdaitsev, and N. Faradzhev, Phys. Solid State 43, 569 (2001).

${ }^{18}$ I. I. Pronin, D. A. Valdaitsev, N. S. Faradzhev, M. V. Gomoyunova, P. Luches, and S. Valeri, Appl. Surf. Sci. 175-176, 83 (2001).

${ }^{19}$ T. S. Kang and J. H. Je, Appl. Phys. Lett. 80, 1361 (2002).

${ }^{20}$ H. Wang, Z. Zhang, L. M. Wong, S. Wang, Z. Wei, G. P. Li, G. Xing, D. Guo, D. Wang, and T. Wu, ACS Nano 4, 2901 (2010).

${ }^{21}$ J. Vrijmoeth, S. Zaima, E. Vlieg, and J. W. M. Frenken, Phys. Rev. B 45, 6700 (1992).

${ }^{22}$ M. G. Wardle, J. P. Goss, P. R. Briddon, and R. Jones, Phys. Status Solidi A 202, 883 (2005).

${ }^{23}$ T. Salditt, T. H. Metzger, and J. Peisl, Phys. Rev. Lett. 73, 2228 (1994).

${ }^{24}$ D. P. Adams, S. M. Yalisove, and D. J. Eaglesham, J. Appl. Phys. 76, 5190 (1994).

${ }^{25}$ See supplementary material at http://dx.doi.org/10.1063/1.3683493 for images of tilted sample and for additional description of the GISAXS procedures, analysis, and modeling. 